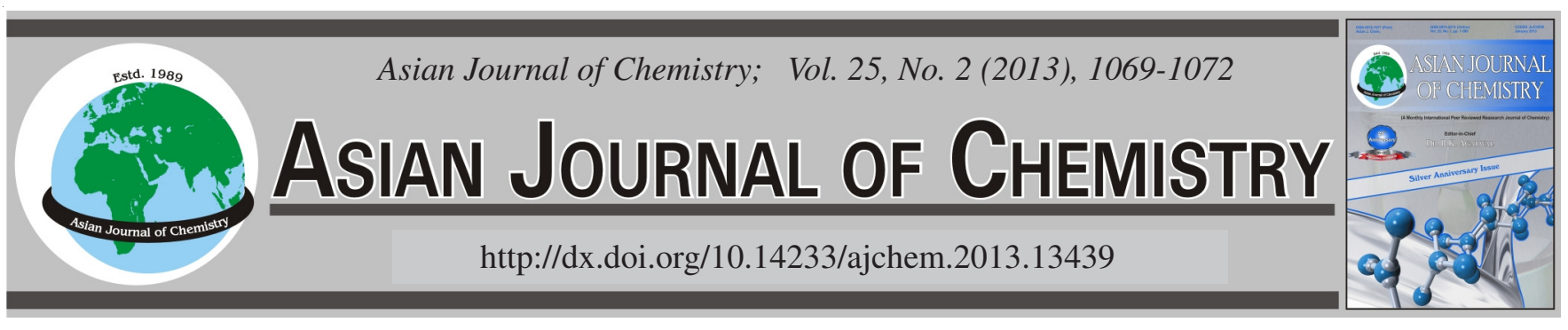

\title{
Antimicrobial Potential and Phytochemical Investigation of Fixed Oil of Plant Chenopodium ambrosioides Linn.
}

\author{
Muhammad Nisar ${ }^{1}$, Hameed Shah ${ }^{1, *}$, Inamullah Khan ${ }^{2}$, Fazal-UR-Rehman $^{3}$, \\ Muhammad Shoaib Khan ${ }^{4}$, Sarfaraz Khan Marwat ${ }^{5}$, Zahid Rasul Niazi ${ }^{3}$ and Aziz Ullah ${ }^{6}$
}

\author{
${ }^{1}$ Institute of Chemical Sciences, University of Peshawar, Peshawar, Pakistan \\ ${ }^{2}$ Department of Pharmacy, University of Peshawar, Peshawar, Pakistan \\ ${ }^{3}$ Faculty of Pharmacy, Gomal University, Dera Ismail Khan, Pakistan \\ ${ }^{4}$ Department of Pharmacy, Islamia University, Bahawalpur, Pakistan \\ ${ }^{5}$ University WENSAM College, Gomal University, Dera Ismail Khan, Pakistan \\ ${ }^{6}$ Department of Microbiology, Kohat University of Science and Technology, Kohat, Pakistan
}

*Corresponding author: E-mail: hameed_shah2002@yahoo.com

\begin{abstract}
Chenopodium ambrosioides is widely used in the traditional medicine system, due to anthelmintic, anticancerous ant antiparasitic activities in Asia, America and Africa. To investigate the fixed oil composition of the plant and evaluate its biological activities, we have selected this medicinal plant for further studies. Twenty four different methyl esters were found in the oil having palmitic acid, linoleic acid, oleic acid, stearic acid, linolenic acid, behenic acid, myristic acid and pentadecanoic acid. The oil also had antimicrobial activity against selected strains of Gram-positive bacteria, Gram-negative bacteria and some fungi species. The results confirm the biological activity of the fixed oil and proved its importance as natural medicinal source.
\end{abstract}

Key Words: Chenopodium ambrosioides, Fixed oil, Methyl esters, Palmitic acid, Stearic acid, Antimicrobial activity.

\section{INTRODUCTION}

Globally infectious diseases are among the factors underlying deaths and are associated with approximately one-half of all mortalities in tropical countries. New, more effective and yet safe therapeutic agents are critical issues to manage challenging infectious diseases ${ }^{1,2}$. Poor communities especially belonging to small isolated villages and native people in developing countries mainly use folk medicine for treatment of infectious diseases ${ }^{2}$. Medicinal plants are consumed as decoctions, teas and juice preparations to treat respiratory infections or as a poultice and applied directly on the infected wounds or burns ${ }^{1,2}$. So far extensive work has been done and still going on for the search of bioactive compounds to treat microbial infections as more effective and safer therapeutic agents.

Chenopodium ambrosioides is an important medicinal plant widely used in the traditional medicine system in Europe, Asia, North and South America. It is a member of family, Chenopodiaceae. This plant is widely distributed in Baluchistan, Peshawar, Dir, Swat, Rawalpindi, Kohala of Pakistan and Kashmir $^{3}$. It is a member of an important plant family,
Chenopodiaceae, which have an elevated importance for phytochemical investigation and medicinal evaluation. Family Chenopodiaceae is consisting of 102 genera and 1400 species $^{4}$. A vast variety of compounds have been reported from the genus Chenopodium. Phytochemically chenopods were reported to contain minerals, primary metabolites-carbohydrates, amino acids, proteins, aromatic cytokinins, hormones, secondary metabolites- such as organic acids, phenol derivatives, lignans, coumarines, flavones, flavonols and their glycosides, flavanones and isoflavones, catechins, sterols, monoterpenes, sesquiterpenoids, triterpenes, carotenoid terpenoides, saponins, alkaloids, amides and amines and also vitamins have been reported from this genus ${ }^{5}$. Oxalic acid has been reported from Chenopodium album in a range of $360-2000 \mathrm{mg} / 100 \mathrm{~g}^{6}$ along with malic acid and succinic acid from Chenopodium ambrosioides ${ }^{7}$. A coumarin scopoletin have been isolated from the aerial parts of Chenopodium murale ${ }^{8}$. Dihydrowogonin, a flavanone as well as the isoflavones irilin A and irilin B were isolated from the aerial parts of $C$. procerum ${ }^{9}$. Saponins isolated from $C$. anthelminticum were tested against influenza type A infections in mouse tissue and were found the strongest antiviral agents ${ }^{10}$. Essential oil from $C$. botrys was examined for their antibacterial 
activities and represent significant action against gram positive and gram negative bacteria ${ }^{11}$. However in literature we could not find any studies on the fixed oil of this important medicinal plant. In our current study we have investigated phytochemical constituents and antimicrobial potential of this plant.

\section{EXPERIMENTAL}

Plant was collected from Peshawar in June and was shade dried. Plant specimen was identified by Dr. Abdur Rashid, Department of Botany, University of Peshawar. Methanolic crude extract of the plant $(10 \mathrm{~kg})$ was further fractionated in $n$-hexane, ethyl acetate, dichloromethane, $n$-butanol and aqueous fractions. Four samples of fixed oil, one from $n$-hexane fraction [at $10 \%$ ethyl acetate and $n$-hexane solvent system by column chromatography (CC)] and three from ethyl acetate fraction (at 1, 3 and $5 \%$ ethyl acetate and $n$-hexane solvent systems by column chromatography) were obtained. Four samples were analyzed by GC-MS for their chemical composition and were tested for antibacterial and antifungal activities.

Gas chromatography-mass spectrometry: All samples were analyzed by GC-MS Model-QP 2010 Plus (Shimadzu). Column Specifications: Length; $30 \mathrm{~mm}$. id; $0.35 \mathrm{~mm}$, thickness; $0.25 \mu \mathrm{M}$, treated with poly ethylene glycol (TRB-FFAP; Technochroma). Injector temperature was $240{ }^{\circ} \mathrm{C}$. Ion source temperature (EI) was $250^{\circ} \mathrm{C}$. The interface temperature was $240^{\circ} \mathrm{C}$. Pressure was maintained at $70 \mathrm{kPa}$. The split ratio was 1:50. Injection volume is $1 \mu \mathrm{L}$ and carrier gas is helium. Identification of the compounds was based on comparison with Wiley and NIST database.

\section{Antibacterial activity}

Agar well diffusion assay: Antibacterial activity was studied via agar well diffusion method. In this method one loop full of $24 \mathrm{~h}$ old culture containing $c a .10^{4}-10^{6} \mathrm{CFU}$ was spread on the surface of Mueller-Hinton Agar plates. Wells were dug in the medium with the help of sterile metallic cork borer. Stock solution of the test samples in the concentration of $22 \mathrm{mg} / \mathrm{mL}$ were prepared in the dimethyl sulphoxide (DMSO) and $150 \mu \mathrm{L}$ dilutions were added in their respective wells. The antibacterial activity of samples was compared with standard drug, streptomycin. The standard drug streptomycin and DMSO were used as positive and negative control. The amount of growth in each well was determined visually by comparing with the growth in the controlled wells.

MIC determination by macrodilution method: Extracts $(10 \mathrm{mg} / \mathrm{mL})$ were dissolved in DMSO and serially diluted with sterile water in microplates in a laminar flow cabinet. The same volume of an actively growing culture of the test bacteria was added to the different wells and cultures were grown overnight in $100 \%$ relative humidity at $378 \mathrm{C}$. Next morning, tetrazolium violet was added to all the wells and the growth was indicated by a violet colour of the culture. Lowest concentration of the test solution that led to inhibition of growth was taken as MIC. Negative control DMSO had no influence on growth even at the highest concentration used.

\section{Antifungal activity}

Antifungal activity was determined by agar well diffusion method. In this method, meconozole was used as the standard drug. Samples were dissolved in DMSO (24 mg/mL). Sterile Sabouraud's dextrose Agar medium ( $7 \mathrm{~mL}$ ) was placed in a test tube and inoculated in a sample solution $(40 \mu \mathrm{g} / \mathrm{mL})$ kept in slanting position at room temperature overnight. Fungal culture was then inoculated on the slant. The samples were incubated for 7 days at $30^{\circ} \mathrm{C}$ and growth inhibition was observed. Per cent growth inhibition was calculated with reference to the negative control by applying the formula:

$(\%)$ Inhibition $=($ Linear growth of the negative control Linear growth of sample) $/ 100 \times 100$

\begin{tabular}{|c|c|c|c|c|c|}
\hline \multicolumn{6}{|c|}{$\begin{array}{l}\text { TABLE-1 } \\
\text { QUANTITATIVE RESULTS OF FATTY ACID METHYL ESTERS OF } n \text {-HEXANE } \\
\text { (10\% ETHYL ACETATE AND HEXANE SOLVENT SYSTEM) SAMPLE BY GC-MS }\end{array}$} \\
\hline Peak\# & Name & Area & Conc. $(\%)$ & Retention time (min) & $\mathrm{m} / \mathrm{z}$ \\
\hline 2. & C8:0; Caprylic acid, methyl ester & 1563 & 0.01 & 4.966 & 87.00 \\
\hline 3. & C10:0; Capric acid, methyl ester & 3598 & 0.03 & 6.802 & 87.00 \\
\hline 4. & C11:0; Undecanoic acid, methyl ester & 1332 & 0.01 & 7.660 & 87.00 \\
\hline 5. & C12:0; Lauric acid, methyl ester & 39792 & 0.35 & 8.555 & 87.00 \\
\hline 6 & C13:0; Tridecanoic acid, methyl ester & 21706 & 0.20 & 9.636 & 87.00 \\
\hline 7. & C14:0; Myristic acid, methyl ester & 124930 & 1.09 & 10.984 & 87.00 \\
\hline 9. & C15:0; Pentadecanoic acid, methyl ester & 144081 & 1.26 & 12.643 & 87.00 \\
\hline 11. & C16:0; Palmitic acid, methyl ester & 5051357 & 44.22 & 14.686 & 87.00 \\
\hline 12. & C16:1c; Palmitoleic acid, methyl ester & 29100 & 0.25 & 15.178 & 97.00 \\
\hline 13. & C17:0; Margaric acid, methyl ester & 145469 & 1.27 & 16.943 & 87.00 \\
\hline 15. & C18:0; Stearic acid, methyl ester & 684728 & 5.99 & 19.006 & 87.00 \\
\hline 16. & C18:1c; Oleic acid, methyl ester & 1209391 & 10.61 & 20.253 & 97.00 \\
\hline 18 & C18:1n8T; Octadecenoic acid, methyl ester & 100267 & 0.88 & 20.451 & 97.00 \\
\hline 19. & C18:2c; Linoleic acid, methyl ester & 2513753 & 22.01 & 21.888 & 95.00 \\
\hline 20 & C18 :2t; Octadecadienoic acid, methyl ester & 12412 & 0.11 & 22.050 & 95.00 \\
\hline 22 & C18:3n3; Linoleic acid, methyl ester & 489959 & 4.29 & 24.364 & 95.00 \\
\hline 23. & C20:0; Arachidic acid, methyl ester & 199210 & 1.74 & 27.068 & 87.00 \\
\hline 24 & C20:1; Eicosenoic acid, methyl ester & 13865 & 0.12 & 27.804 & 97.00 \\
\hline 27. & C21:0; Heneicosanoic acid, methyl ester & 29418 & 0.26 & 30.878 & 87.00 \\
\hline 31. & C22:0; Behenic acid, methyl ester & 275888 & 2.42 & 34.335 & 87.00 \\
\hline 34. & C23:0; Tricosanoic acid, methyl ester & 96202 & 0.84 & 37.586 & 87.00 \\
\hline 35. & C24:0; Tetracosanoic acid, methyl ester & 230443 & 2.02 & 40.675 & 87.00 \\
\hline
\end{tabular}




\section{RESULTS AND DISCUSSION}

Four samples of fixed oil obtained from chenopodium ambrosioides linn were analyzed by GC MS. Results obtained from the GC-MS of fixed oil of this plant along with the GC-MS of 37 components used as standard are given below. Total 22 components (Table-1) were identified from sample 1 by GC-MS. Important components in highest concentration were palmitic acid $(44.22 \%)$, linoleic acid (22.01\%), oleic acid $(10.61 \%)$, stearic acid $(5.99 \%)$, linolenic acid (4.29\%) and behenic acid $(2.42 \%)$ while the other components were in the small quantity. In case of compounds in sample 2 (Table-2), major components were palmitic acid (62.41\%), stearic acid (18.31\%), myristic acid (4.33\%), pentadecanoic acid (3.97\%) and behenic acid $(3.37 \%)$ apart from minor components. Similarly palmitic acid $(66.68 \%)$, stearic acid $(8.19 \%)$, oleic acid $(8.11 \%)$, tetracosanoic acid $(2.56 \%)$ and arachidic acid $(2.35 \%)$ are the major components of sample 3 (Table-3) while the others are present in minor quantities. major compounds identified in sample 4 (Table-4) are palmitic acid (61.66 \%), stearic acid $(11.75 \%)$, pentadecanoic acid $(7.92 \%)$, oleic acid $(4.92 \%)$ and myristic acid $(3.57 \%)$ while the others are present in minor quantities.
Antimicrobial activities: Significant antibacterial activity was found against a set of various human pathogens including Escherichia coli, Klebsiella pneumoniae (Gram negative bacteria), Staphylococcus aureus, Bacilus subtilus and Staphylococcus epidermidis (Gram positive bacteria). The most susceptible tested bacterial strain was Staphylococcus aureus against sample 4 followed by sample 2 . In all cases oily fraction showed moderate but significant activities (Tables 5 and 6). Similarly good results were obtained against Escherichia coli as well. In case of antifungal activities (Table-7), no considerable results were found against tested fungal strains. Only low activity profile was found incase of sample 1 and 2 against various fungi. These inspiring results highlighted the strong potential of fixed to identify chemical constituents that might be involved in antibacterial action followed by mechanistic studies to explore their molecular targets.

\section{ACKNOWLEDGEMENTS}

Deep thanks and grateful acknowledgments are due to Prof. Dr. Abdur Rashid, Department of Botany, University of Peshawar, Pakistan.

TABLE-2

QUANTITATIVE RESULTS OF FATTY ACID METHYL ESTER OF ETHYL ACETATE FRACTION ( $1 \%$ ETHYL ACETATE AND HEXANE SOLVENT SYSTEM) SAMPLE BY GC-MS

\begin{tabular}{clcccc}
\hline Peak\# & \multicolumn{1}{c}{ Name } & Area & Conc. (\%) & Retention time (min) & $\mathrm{m} / \mathrm{z}$ \\
\hline 5. & C12:0; Lauric acid, methyl ester & 2414 & 1.19 & 8.553 & 87.00 \\
7. & C14:0; Myristic acid, methyl ester & 8784 & 4.33 & 10.982 & 87.00 \\
9. & C15:0; Pentadecanoic acid, methyl ester & 8060 & 3.97 & 12.640 & 87.00 \\
11. & C16:0; Palmitic acid, methyl ester & 126636 & 62.41 & 14.632 & 87.00 \\
13. & C17:0; Margaric acid, methyl ester & 7190 & 3.54 & 16.935 & 87.00 \\
15. & C18:0; Stearic acid, methyl ester & 37154 & 18.31 & 19.632 & 87.00 \\
31. & C22:0; Behenic acid, methyl ester & 6830 & 3.37 & 34.331 & 87.00 \\
32. & C22:1; Eruccic acid, methyl ester & 5849 & 2.88 & 34.903 & 97.00 \\
\hline
\end{tabular}

TABLE-3

QUANTITATIVE RESULTS OF FATTY ACID METHYL ESTERS OF ETHYL ACETATE ( $3 \%$ ETHYL ACETATE AND HEXANE SOLVENT SYSTEM) SAMPLE BY GC-MS

\begin{tabular}{|c|c|c|c|c|c|}
\hline Peak\# & Name & Area & Conc. (\%) & Retention time (min) & $\mathrm{m} / \mathrm{z}$ \\
\hline 1 & C6:0; Hexanoic acid, methyl ester & 55302 & 1.30 & 3.069 & 87.00 \\
\hline 2 & C8:0; Caprylic acid, methyl ester & 4971 & 0.12 & 4.967 & 87.00 \\
\hline 3 & C10:0; Capric acid, methyl ester & 2501 & 0.06 & 6.803 & 87.00 \\
\hline 4 & C11:0; Undecanoic acid, methyl ester & 578 & 0.01 & 7.658 & 87.00 \\
\hline 5 & C12:0; Lauric acid, methyl ester & 10064 & 0.24 & 8.555 & 87.00 \\
\hline 6 & C13:0; Tridecanoic acid, methyl ester & 3157 & 0.20 & 9.638 & 87.00 \\
\hline 7 & C14:0; Myristic acid, methyl ester & 52592 & 1.24 & 10.985 & 87.00 \\
\hline 9 & C15:0; Pentadecanoic acid, methyl ester & 68563 & 1.61 & 12.645 & 87.00 \\
\hline 11. & C16:0; Palmitic acid, methyl ester & 2837158 & 66.68 & 14.665 & 87.00 \\
\hline 12. & C16:1c; Palmitoleic acid, methyl ester & 8947 & 0.21 & 15.181 & 97.00 \\
\hline 13. & C17:0; Margaric acid, methyl ester & 61259 & 1.44 & 16.940 & 87.00 \\
\hline 15. & C18:0; Stearic acid, methyl ester & 348324 & 8.19 & 19.645 & 87.00 \\
\hline 16. & C18:1c; Oleic acid, methyl ester & 345228 & 8.11 & 20.198 & 97.00 \\
\hline 18 & C18:1n8T; Octadecenoic acid, methyl ester & 23847 & 0.56 & 20.432 & 97.00 \\
\hline 19. & C18:2c; Linoleic acid, methyl ester & 45603 & 1.07 & 21.777 & 95.00 \\
\hline 27. & C21:0; Heneicosanoic acid, methyl ester & 10115 & 0.24 & 30.885 & 87.00 \\
\hline 31. & C22:0; Behenic acid, methyl ester & 133278 & 3.13 & 34.338 & 87.00 \\
\hline 34. & C23:0; Tricosanoic acid, methyl ester & 37746 & 0.89 & 37.596 & 87.00 \\
\hline 35. & C24:0; Tetracosanoic acid, methyl ester & 108992 & 2.56 & 40.684 & 87.00 \\
\hline
\end{tabular}




\begin{tabular}{|c|c|c|c|c|c|}
\hline \multicolumn{6}{|c|}{$\begin{array}{l}\text { TABLE-4 } \\
\text { QUANTITATIVE RESULTS OF FATTY ACID METHYL ESTERS OF ETHYL ACETATE } \\
\text { (5\% ETHYL ACETATE AND HEXANE SOLVENT SYSTEM) SAMPLE BY GC-MS }\end{array}$} \\
\hline Peak\# & Name & Area & Conc. $(\%)$ & Retention time (min) & $\mathrm{m} / \mathrm{z}$ \\
\hline 1. & C6:0; Hexanoic acid, methyl ester & 2125 & 0.59 & 3.068 & 87.00 \\
\hline 2. & C8:0; Caprylic acid, methyl ester & 1127 & 0.31 & 4.966 & 87.00 \\
\hline 3. & C10:0; Capric acid, methyl ester & 1576 & 0.44 & 6.805 & 87.00 \\
\hline 5. & C12:0; Lauric acid, methyl ester & 5275 & 1.47 & 8.557 & 87.00 \\
\hline 6. & C13:0; Tridecanoic acid, methyl ester & 1535 & 0.20 & 9.310 & 87.00 \\
\hline 7. & C14:0; Myristic acid, methyl ester & 12797 & 3.57 & 10.983 & 87.00 \\
\hline 9. & C15:0; Pentadecanoic acid, methyl ester & 28435 & 7.92 & 12.657 & 87.00 \\
\hline 11. & C16:0; Palmitic acid, methyl ester & 221256 & 61.66 & 14.634 & 87.00 \\
\hline 13. & C17:0; Margaric acid, methyl ester & 6015 & 1.68 & 16.935 & 87.00 \\
\hline 15. & C18:0; Stearic acid, methyl ester & 42153 & 11.75 & 19.634 & 87.00 \\
\hline 16. & C18:1c; Oleic acid, methyl ester & 17664 & 4.92 & 20.175 & 97.00 \\
\hline 19. & C18:2c; Linoleic acid, methyl ester & 11373 & 3.17 & 21.773 & 95.00 \\
\hline 31. & C22:0; Behenic acid, methyl ester & 9064 & 2.53 & 34.334 & 87.00 \\
\hline
\end{tabular}

\section{TABLE-5}

DIAMETER OF ZONE OF INHIBITION (mm) OF SAMPLES AGAINST BACTERIAL STRAINS

\begin{tabular}{|c|c|c|c|c|c|}
\hline Sample No. & Escherichia coli & $\begin{array}{c}\text { Klebsiella } \\
\text { pneumoniae }\end{array}$ & $\begin{array}{c}\text { Staphylococcus } \\
\text { aureus }\end{array}$ & Bacilus subtilus & $\begin{array}{c}\text { Staphylococcus } \\
\text { epidermidis }\end{array}$ \\
\hline 1 & 10 & 12 & 12 & 16 & 10 \\
\hline 2 & 10 & 08 & 14 & 00 & 12 \\
\hline 3 & 10 & 12 & 12 & 10 & 12 \\
\hline 4 & 16 & 10 & 14 & 10 & 00 \\
\hline DMSO & 00 & 00 & 00 & 00 & 00 \\
\hline Streptomycin & 26 & 28 & 26 & 30 & 30 \\
\hline
\end{tabular}

TABLE-6

MINIMUM INHIBITORY CONCENTRATIONS (MIC: $\mu \mathrm{g} / \mathrm{mL}$ ) OF TEST SAMPLES

\begin{tabular}{|c|c|c|c|c|c|}
\hline \multirow[b]{2}{*}{ Sample No. } & \multicolumn{5}{|c|}{ Minimum inhibitory concentrations $(\mu \mathrm{g} / \mathrm{mL})$} \\
\hline & Escherichia coli & $\begin{array}{c}\text { Klebsiella } \\
\text { pneumoniae }\end{array}$ & $\begin{array}{c}\text { Staphylococcus } \\
\text { aureus }\end{array}$ & Bacilus subtilus & $\begin{array}{c}\text { Staphylococcus } \\
\text { epidermidis }\end{array}$ \\
\hline 1 & $0.6 \pm 0.05$ & $0.4 \pm 0.02$ & $0.40 \pm 0.03$ & $0.40 \pm 0.05$ & $0.6 \pm 0.02$ \\
\hline 2 & $0.6 \pm 0.06$ & $0.9 \pm 0.04$ & $0.2 \pm 0.02$ & - & $0.4 \pm 0.01$ \\
\hline 3 & $0.6 \pm 0.02$ & $0.4 \pm 0.01$ & $0.4 \pm 0.03$ & $0.6 \pm 0.05$ & $0.4 \pm 0.04$ \\
\hline 4 & $0.2 \pm 0.04$ & $0.6 \pm 0.02$ & $0.1 \pm 0.02$ & $0.6 \pm 0.02$ & - \\
\hline Streptomycin & $0.02 \pm 0.001$ & $0.06 \pm 0.001$ & $0.03 \pm 0.04$ & $0.014 \pm 0.001$ & $0.09 \pm 0.003$ \\
\hline
\end{tabular}

Sample No. $1=n$-Hexane $(10 \%), 2=$ Ethyl acetate $(1 \%), 3=$ ethyl acetate $(3 \%), 4=$ Ethyl acetate $(5 \%)$

\section{TABLE-7}

PERCENTAGE INHIBITION (mm) OF SAMPLES AGAINST FUNGAL STRAINS

\begin{tabular}{cccccc}
\hline Sample No & Aspergillus niger & $\begin{array}{c}\text { Aspergillus } \\
\text { parasiticus }\end{array}$ & $\begin{array}{c}\text { Trycophyton } \\
\text { horzianum }\end{array}$ & Rizopus tolenapur & Aspergillus flavus \\
\hline 1 & 36 & 35 & 15 & 31 & 30 \\
2 & 25 & 36 & 00 & 30 & 10 \\
3 & 26 & 22 & 10 & 00 & 00 \\
4 & 00 & 20 & 30 & 18 & 10 \\
Miconazole & 100 & 100 & 100 & 100 & 100 \\
\hline Sample No. $1=n$-Hexane $(10 \%), 2$ Ethyl acetate $(1 \%), 3=$ Ethyl acetate $(3 \%), 4$ = Ethyl acetate $(5 \%)$ &
\end{tabular}

\section{REFERENCES}

1. J. Gonzalez, J. Ethnopharmacol., 2, 43 (1980).

2. M. Nisar, I. Khan, B. Ahmad, I. Ali, W. Ahmad and M.I. Choudhary, J. Enzyme Inhib. Med. Chem., 23, 256 (2008).

3. Herbarium S. Flora of West Pakistan (1972).

4. C.N. Marie, In Gardens of Hawaii, Bishop Museum Press, p. 331 (1965).

5. Z. Kokanova-Nedialkova, P.T. Nedialkov and S.D. Nikolov, Phcog. Rev., 3, 280 (2009)

6. J.L. Guil, M.E. Torija, J.J. Gimenez, I.R. Garcia and A. Gimenez, J. Agric. Food Chem., 44, 1821 (1996).
7. G.S. Gupta, N. Lal and M. Behari, Proc. Nat. Acad. Sci. India, 41A, 1 (1971).

8. N.H. El-Sayed, A.S. Awaad, M.S. Hifnawy and T.J. Mabry, Phytochemistry, 51, 591 (1999).

9. C. Bergeron, A. Marston, E. Hakizamungu and K. Hostettmann, Int. J. Pharmacog., 33, 115 (1995).

10. Z.A. Maksimovic, S. Dordevic and M. Mraovic, Fitoterapia, 76, 112 (2005).

11. O. Tzakou, A. Pizzimenti, F.C. Pizzimenti, V. Sdrafkakis and E.M. Galati, J. Essent. Oil Res., 19, 292 (2006). 